\title{
Goodman And Parry on Counterfactuals
}

\author{
OSWALDO CHATEAUBRIAND \\ PUC-Rio/CNPq
}

\begin{abstract}
Goodman's paper "The Problem of Counterfactual Conditionals" played a central role in the debate concerning the proper analysis of counterfactual conditionals. In what follows I examine Goodman's paper in detail and discuss objections and suggestions by Parry in his "Reexamination of the Problem of Counterfactual Conditionals". I restrict my discussion to what Goodman termed "the problem of relevant conditions", which is the main subject of Parry's criticism, and which I also consider to be the central issue for Goodman's approach.
\end{abstract}

Keywords: N. Goodman; W. T. Parry; counterfactual conditionals; relevant conditions.

\section{Goodman's analysis}

Goodman summarizes his basic view of counterfactuals as follows (1947, p. 7-9):

As I see it, there are two major problems, though they are not independent and may even be regarded as aspects of a single problem. A counterfactual is true if a certain connection obtains between the antecedent and the consequent. But as is obvious from examples already given, the consequent seldom follows from the antecedent by logic alone. (1) In the first place, the assertion that a connection holds is made on the presumption that certain circumstances not stated in the antecedent obtain. When we say

If that match had been scratched, it would have lighted, we mean that conditions are such - i.e., the match is well made, is dry enough, oxygen enough is present, etc. - that "That match lights" can be inferred from "That match is scratched". Thus the connection we affirm may be regarded as joining the consequent with the conjunction of the antecedent with other statements that truly describe relevant conditions. ... The first major problem is to define relevant conditions: to specify what sentences are meant to be taken in conjunction with the antecedent as a basis for inferring the consequent. (2) But even after the particular relevant conditions are specified, the connection obtaining will not ordinarily be a logical law... but what we call a natural or physical or causal law. The second major problem concerns the definition of such laws.

Relevant conditions are described by true sentences, but obviously not all true sentences can be taken as describing relevant conditions. For, otherwise, since the negation of the antecedent of any counterfactual is true, all counterfactuals would

Principia 15(3): 383-397 (2011).

Published by NEL — Epistemology and Logic Research Group, Federal University of Santa Catarina (UFSC), Brazil. 
be true by Goodman's formula. By the same token, also those sentences from which the negation of the antecedent of a given counterfactual follows by law cannot be taken as describing relevant conditions for that counterfactual. One must require, therefore, that the sentences describing relevant conditions for a counterfactual bear some relation to its antecedent. A minimal requirement is that they be compatible with the antecedent; i.e., that jointly with the antecedent they should not lead by law to a contradiction. We thus have, as a first step:

(1.1) A counterfactual is true if the consequent follows by law from the antecedent together with a set of true sentences compatible with the antecedent.

But Goodman quickly points out that further requirements are needed, offering the following example:

(1.2) If Jones were in Carolina, he would be in North Carolina,

(1.3) If Jones were in Carolina, he would be in South Carolina.

Assuming Jones is not in Carolina, both counterfactuals are true according to (1.1). For, as relevant conditions for (1.2) we can take the sentences "North Carolina plus South Carolina is identical to Carolina" and "Jones is not in South Carolina", and as relevant conditions for (1.3) we can take the sentences "North Carolina plus South Carolina is identical to Carolina" and "Jones is not in South Carolina".

This leads Goodman to the more elaborate formulation:

(1.4) A counterfactual $A>C$ is true if

[a] there is a sentence $S$ such that

(1) $S$ is true,

(2) $S$ is compatible with $C$ and with $\neg C$,

(3) $S$ is compatible with $A$,

(4) $A \& S$ leads by law to $C$,

and [b] there is no sentence $S^{\prime}$ such that

$\left(1^{\prime}\right) S^{\prime}$ is true,

(2') $S^{\prime}$ is compatible with $C$ and with $\neg C$,

(3') $S^{\prime}$ is compatible with $A$,

$\left(4^{\prime}\right) \quad A \& S^{\prime}$ leads by law to $\neg C$.

The main difference between this formulation and (1.1) is the addition of clause [b], which is essentially a consistency condition: if two counterfactuals with the same antecedent and incompatible consequents are both accepted by (1.1), then

Principia 15(3): 383-397 (2011). 
neither is true. The remaining additions to (1.1) are mostly inessential. That is, except for the requirement that $S^{\prime}$ be compatible with $C$, the conditions in (2) and $\left(2^{\prime}\right)$ are redundant.

From (3) and (4) it follows that $S$ is compatible with $C$, for, otherwise, $\neg C$ follows by law from $S$, and, by (4), $A \& S$ leads by law to $C \& \neg C$, contradicting (3). Similarly, from $\left(3^{\prime}\right)$ and $\left(4^{\prime}\right)$ it follows that $S^{\prime}$ is compatible with $\neg C$. Furthermore, from (1) and the falsity of $C$, it follows that $S$ and $\neg C$ are both true, and so $a$ fortiori compatible. The compatibility of $S^{\prime}$ with $C$ cannot be derived from the other conditions and is needed for the same reasons given in favor of (3). Specifically, if $S^{\prime}$ were not required to be compatible with $C$, then for any counterfactual such that $C$ does not follow by law from $A$ alone (i.e., such that $\neg C$ is compatible with $A$ ), $\neg C$ would be eligible as $S^{\prime}$ and [b] would not be satisfied.

Although (1.4) seems to take care of the problem raised by the counterfactuals (1.2) and (1.3), it is also immediately rejected by Goodman on the grounds that, for a normal and dry match $m$ in ordinary circumstances, it does not discriminate between the counterfactuals

(1.5) If $m$ had been scratched, it would have lighted,

and

(1.6) If $m$ had been scratched, it would not have been dry.

He claims that, ordinarily, we would assert (1.5) and deny (1.6). But if (1.5) is supported by a general principle of the form

(1.7) For every match $x$, if $x$ is well made, $x$ is dry enough, etc., and $x$ is scratched, then $x$ lights,

plus the conditions that $m$ is well made, is dry enough, etc., then (1.6) is equally supported by the contrapositive

(1.8) For every match $x$, if $x$ is well made, $x$ does not light, etc., and $x$ is scratched, then $x$ is not dry,

plus the conditions that $m$ is well made, $m$ did not light, etc.

Goodman's diagnosis at this point is that the condition that $m$ did not light used to support (1.6), although compatible with the antecedent of (1.6), is such that it would not be true if the antecedent were true - this, presumably, is so because (1.5) is true. He proposes then to modify (1.4) by strengthening the requirements of compatibility in (3) and ( $\left.3^{\prime}\right)$ to requirements of cotenability; where $A$ is said to be cotenable with $S$ if the counterfactual

If $A$ were the case, then $S$ would not be the case,

Principia 15(3): 383-397 (2011). 
is false. But this, of course, is obviously circular.

Although Goodman has emphasized this difficulty, and it has become the starting point of most discussions of his paper, there are a number of other important features of his analysis that have not been satisfactorily understood. I will consider some of these before turning to the question of cotenability.

\section{Parry's analysis}

Parry (1957, p. 86) pointed out that the conditions stated in (1.4) can be easily circumvented by using as $S$ and $S^{\prime}$ the material conditionals $A \rightarrow C$ and $A \rightarrow \neg C$, respectively. The problem can be stated more precisely as follows:

(2.1) A counterfactual $A>C$ is true by (1.4) if and only if $A$ is compatible with $C$ and either $A$ leads by law to $C$ or $C$ leads by law to $A$.

Note first that by (3) of (1.4) all counterfactuals with self-incompatible antecedent are false; i.e., as Goodman points out, all counterlegals are false. We see also that by (3) and (4) all counterfactuals such that $A$ is incompatible with $C$ are false. Granted the exclusion of counterlegals, this seems as it should be. What is surprising is that this is all that is achieved by clause [a]; i.e., any counterfactual such that

(2.2) $A$ is compatible with $C$,

satisfies clause [a]. Let $S$ be the sentence $A \rightarrow C$. Since $A$ is false this sentence is true. It is compatible with $A$ because otherwise $A$ would lead by law to $\neg(A \rightarrow C)$, and, hence, to $\neg C$, contradicting (2.2). Finally, $A \&$ ( $A$ to $C$ ) implies $C$.

Of the counterfactuals satisfying (2.2) let us consider now those that might be said to be the simplest kind of true conterfactuals; namely, those such that

$$
C \text { follows by law from } A \text { alone. }
$$

These counterfactuals are true by any reasonable intuitive standards, and they are true by (1.4), because any $S^{\prime}$ satisfying $\left(4^{\prime}\right)$ will contradict ( $\left.3^{\prime}\right)$, violating clause [b].

Up to this point (1.4) has been reasonably well behaved; extensionally, at any rate. It is for the remaining counterfactuals - which include the interesting cases - that it goes awry. Indeed, also the counterfactuals satisfying (2.2) and

(2.4) $A$ follows by law from $C$ alone,

are all true. To see that [b] is fulfilled, assume that there is an $S^{\prime}$ satisfying $\left(1^{\prime}\right)$ $\left(4^{\prime}\right)$. By $\left(4^{\prime}\right), A \& S^{\prime}$ leads by law to $\neg C$, and, using (2.4) $S^{\prime} \& C$ leads by law to $\neg C$, contradicting $\left(2^{\prime}\right)$. But, of course, counterfactuals satisfying (2.4) are typically false.

Finally, all other counterfactuals are false according to (1.4). For, assume that

Principia 15(3): 383-397 (2011). 
(2.5) $\neg A$ is compatible with $C$,

and

(2.6) $A$ is compatible with $\neg C$,

and let $S^{\prime}$ be $A \rightarrow \neg C$. $S^{\prime}$ is true since $A$ is false. It is compatible with $C$ by (2.5) and with $A$ by (2.6). This is again wrong because many true counterfactuals satisfy conditions (2.5) and (2.6).

Some of what is wrong with (1.4) is quite obvious. Briefly, for the same reasons that $\neg A$ must be excluded as a relevant condition, also $A \rightarrow C$ and $A \rightarrow \neg C-$ whose truth, in our use of them, depended on $\neg A$ being true - should be excluded. More generally, the problem is to rule out such illegitimate information that depends on the actual facts denied by the counterfactual assumption but which is not actually incompatible with it. Both Goodman and Parry suggest revisions along these lines and I will review their proposals next.

\section{Goodman's proposal}

Following up on some ideas of Parry, Goodman (1957, p. 444) proposes to avoid the previous objections by adding to (1.4) that neither $S$ nor $S^{\prime}$ follow by law from $\neg A$. That is, he adds

(5) $\neg A$ is compatible with $\neg S$,

and

$\left(5^{\prime}\right) \quad \neg A$ is compatible with $\neg S^{\prime}$.

Although these conditions effectively bar $A \rightarrow C$ and $A \rightarrow \neg C$ as choices for $S$ and $S^{\prime}$, it is easy to modify the arguments so that all the above results remain valid. For any counterfactual such that $A$ is compatible with $C$, let $Q$ be any sentence satisfying the following conditions:

(3.1) $Q$ is true,

(3.2) $Q$ does not follow by law from $\neg A$,

(3.3) $Q$ is compatible with $A$,

(3.4) $Q$ is compatible with $C$.

It is obvious that the preceding arguments can be repeated almost verbatim using $(A \rightarrow C) \& Q$ and $(A \rightarrow \neg C) \& Q$ in place of $A \rightarrow C$ and $A \rightarrow \neg C$. Of course, (5) and $\left(5^{\prime}\right)$ are satisfied in virtue of (3.2), and we can always find such sentences $Q$, for irrelevant true sentences will do. To deal with any of the counterfactuals mentioned

Principia 15(3): 383-397 (2011). 
so far, or any others in Goodman's book, we may take as $Q$ the sentence "Socrates died in 399 B.C."

Another feature of Parry's examples that attracts attention is that they are logical tricks. One might consider adding to (1.4), besides (5) and $\left(5^{\prime}\right)$ :

$$
A \& S \text { does not imply } C \text {, }
$$

and

$\left(6^{\prime}\right) \quad A \& S^{\prime}$ does not imply $\neg C$.

These conditions would exclude some legitimate counterfactuals, but even if we can deal with these separately, the status of the other counterfactuals is not changed. For, let $Q$ be as before and let $P$ and $P^{\prime}$ be such that:

(3.5) $P$ leads by law to $C$ but does not imply $C$,

(3.6) $P^{\prime}$ leads by law to $\neg C$ but does not imply $\neg C$.

The arguments will go through again using $(\neg A \& Q) \vee(C \& Q) \vee(P \& Q)$ as $S$, and $(\neg A \& Q) \vee(\neg C \& Q) \vee\left(P^{\prime} \& Q\right)$ as $S^{\prime}$. Since $P$ and $P^{\prime}$ need not be true, it is reasonable to assume that they can be found in most, if not all, specific cases.

Since it seems clear that this kind of maneuvers will not do, let me examine now Parry's more interesting suggestions.

\section{Parry's proposals}

Parry argues that since Goodman's addition of clause [b] bars many true counterfactuals, it should be dropped altogether. He proposes to strengthen [a], and to this end he reexamines Goodman's motivation for introducing [b] - i.e., examples (1.2) and (1.3) - and adduces the following considerations (p. 90):

Now, when we begin "If Jones were in Carolina, ..." we are abstracting from his actual geographic location, and putting him in a presumably non-actual location, while leaving other things, as far as possible, the same. It is plain that the "other things" which may be included in the relevant conditions, $S$, must not include anything about his actual location at the time of the antecedent. (Note: Of course we do not want to exclude possibly relevant information about his location at other times, provided it does not "give away" his present location.) This plain fact is never mentioned by Goodman, whether because it is too vague, too special, or for another reason. I will try to give a formal explication of this restriction, but do not guarantee it is fully adequate.

Principia 15(3): 383-397 (2011). 
To this Goodman replies (1957, p. 444) that the plain fact "is surely too special since it applies only to counterfactuals concerning location; and is also too vague in view of the extreme fuzziness of 'about'." This does not detract from its plainness though, but only from its formal usefulness in a definition.

Parry attempts to explain the above restriction more generally and formally by adding to [a], instead of (5), a stronger condition $\left(5^{\#}\right)$, which reads:

$\left(5^{\#}\right) \quad S$ is not tautologically equivalent to any truth-function containing as essential component a proposition to which (or the negation of which) $\neg A$ leads by law.

Similarly, he strengthens condition (3) to:

$\left(3^{\#}\right) \quad S$ is not tautologically equivalent to any truth-function containing as essential component a proposition which (or the negation of which) leads by law to $\neg A$.

Concerning the notion "essential component", Parry offers the following explanation (1957, p. 91):

(4.1) We say "essential component" because any $S$ is equivalent to $(S \& A) \vee(S \&$ $\neg A$ ), in which $\neg A$ is a component to which $\neg A$ leads by law; but $A, \neg A$ are not essential components of the cited truth-function, being eliminated in the equivalent statement $S$.

If one looks back to the arguments in sections 2 and 3 above, Parry's moves appear to be both natural, because we do want the relevant conditions to be independent of the falsity of the antecedent, and efficient, because at the crucial steps in these arguments we did violate Parry's restrictions on essential components. But with respect to examples (1.2) and (1.3) Goodman argues that Parry's explication does not exclude all sentences containing information about Jones' actual location, and that these counterfactuals can both be true according to Parry's revised formulation. For, assume that at the time in question in (1.2) and (1.3) Jones was in South Dakota, and consider the sentences:

(4.2) Jones is in a state north of South Carolina,

and

(4.3) Jones is in a state whose name contains the word "South".

From what we have said so far these statements do not seem to be excluded as choices for conjuncts of $S$, but since they obviously carry information as to Jones' actual location, Parry's explication of his informal requirement is inadequate. Furthermore, using (4.2), (4.3) and some additional sentences it can be shown that (1.2) and (1.3) are both true according to Parry's formulation.

Principia 15(3): 383-397 (2011). 
Although Parry's formal explication is thus shown to be inadequate, we cannot conclude from this that his informal requirement - which is violated by Goodman's examples - is also inadequate. I will return to this issue later, but first I want to consider Parry's more specific claim that clause [b] should be dropped.

\section{Arguments against clause [b]}

Parry argues (p. 86) that clause [b] should be dropped because, since $A \rightarrow \neg C$ is eligible as $S^{\prime}$, it bars many true counterfactuals. This is not very convincing, however. The eligibility of $A \rightarrow \neg C$ is not due to clause [b] itself but, rather, to the formulation of the basic conditions in [a]. One might agree that [a] needs strengthening and yet believe that a consistency clause like [b] is the right way of meeting the problem raised by such counterfactuals as (1.2) and (1.3), which seems to be Goodman's view. I will argue that this is very unlikely, and that, independently of the formulation of $[\mathrm{a}]$, the idea behind the formulation of [b] is essentially misguided.

I assume there is a distinction between those facts, or sentences - the relevant conditions - that can legitimately be used in support of a counterfactual and those that cannot. I think Parry is right in suggesting that the antecedent of (1.2) and (1.3) involves abstracting upon Jones' actual location and that any information concerning this location should be illegitimate. It is, in fact, rather surprising that Goodman never pauses to consider what distinguishes the legitimate from the illegitimate information. However vague the criteria may be, it is just what one should be after. For, if there is a distinction, then the problem of relevant conditions is to characterize it; and if there isn't, then Goodman's approach loses its appeal because one will only get a good definition by sheer luck. Let us assume, therefore, that there is a distinction between legitimate and illegitimate information and that it is permissible to talk about all legitimate information for a given counterfactual—where, obviously, the "given" includes context.

Consider now the counterfactuals (1.2) and (1.3). That they cannot be both true in the same context is clear. It is also clear that the context Goodman imagines is one in which they are both false, and that he would agree that such information as "Jones is not in South Carolina" and "Jones is not in North Carolina" is illegitimate. But how does Goodman handle this situation? He allows the illegitimate information to slip in and rules out both counterfactuals by clause [b]. It should be obvious, however, that this cannot work in general, because if the definition allows this illegitimate information to slip in in this case, it will not be able to bar it in other cases (or contexts) in which one of the counterfactuals is actually true. For, although these counterfactuals cannot be both true, they need not be both false. If Jones lives in North Carolina close to the Virginia border and commutes to work in Virginia, one

Principia 15(3): 383-397 (2011). 
can easily imagine circumstances in which (1.2) might be true and (1.3) false.

This can be generalized to a plausibility argument against a clause like [b]. For, if [b] is needed at all, it is because the conditions in [a] do not bar all illegitimate information - this depends on the assumption that all legitimate information together with the antecedent cannot lead to incompatible consequents. So, suppose we have a pair of counterfactuals with the same antecedent $A$ and incompatible consequents $C$ and $C^{\prime}$ that the definition classifies as false by essential use of clause [b]. This means that there are $S$ and $S^{\prime}$ satisfying whatever conditions are included in [a], which together with $A$ lead by law to $C$ and $C^{\prime}$, respectively. (Of course, if the counterfactuals are classified as false because there are no acceptable $S$ and $S^{\prime}$, this would be independent of clause [b].) Suppose, moreover, that both $S$ and $S^{\prime}$ contain illegitimate information - since at least one of them does, if the other does not, this would already constitute a counterexample to [b]. Now, I suggest it is plausible that in any such case there is another situation in which one of the counterfactuals is actually true - and, hence, there is legitimate information that establishes it and such that the information used for the other counterfactual is still true but illegitimate. Although I think it is likely that this strong claim is true, all that is needed to refute $[\mathrm{b}]$ is the existence of some cases of this kind.

A second problem, somewhat complementary to the previous one, is the following. Suppose we have two counterfactuals with the same antecedent $A$ and incompatible consequents $C$ and $C^{\prime}$ which are actually false. Suppose, moreover, that the conditions in [a] are satisfied for one of them by use of illegitimate information. What reason is there to suppose that in any such case there will also be illegitimate information that allows the other counterfactual to satisfy the conditions of [a], and, hence, to rule both out by [b]? Whereas the first problem raises a question concerning the soundness of [b] for certain true counterfactuals, this raises it for certain false counterfactuals. A more specific problem that raises the same question is the following.

As can be seen from the part of my argument in section 2 concerning those counterfactuals that satisfy (2.4), clause [b] is partly to blame here too - and the same problem arises if instead of $\neg C$ one uses a $C^{\prime}$ incompatible with $C$. Of course, a strengthening of [a] may bar many of the false counterfactuals satisfying (2.4), but if some are let through, and $\left(4^{\prime}\right)$ and $\left(2^{\prime}\right)$ are necessary conditions, then the problem arises again. This point can also be generalized, for a special case, to a plausibility argument against [b] more or less independent of the formulation of [a]. Intuitively, if a counterfactual $A>C$ is true, so is the counterfactual $A>A \& C$. Suppose the conditions in [a] also have this property, and consider a false counterfactual $A>C$ that gets through [a] by means of illegitimate information. Then, even if a clause like [b] bars it by means of some appropriate $C^{\prime}$, by the preceding considerations it will not be able to bar the counterfactual $A>A \& C$, which is also false. 
Although I have not proved my point, I think I have made a sufficiently strong case that a proponent of a clause like [b] should have to give convincing reasons for the soundness of its adoption.

It may be suggested that the problem lies not so much with the idea of a consistency clause but with the specific formulation chosen by Goodman. I do not think so, for the natural alternatives I have considered are faced with some, if not all, of the same problems. An example may help clarify the issue a bit further.

Suppose (1) through $(n)$ are necessary conditions, including truth and compatibility with $A$, for sentences being relevant conditions. Say that a set of sentences $\Sigma$ is maximal with respect to $(1)-(n)$ if it satisfies $(1)-(n)$ and no extension of it does. One might then say:

(5.1) A counterfactual $A>C$ is true if for any $\Sigma$ that is maximal with respect to (1)-(n), $A$ together with $\Sigma$ leads by law to $C$.

I am assuming, as with Goodman's original formulation, that this form of definition is needed because (1)-(n) are not sufficient to bar all illegitimate information. Otherwise we could simply say: there is a $\Sigma$ such that (1)-(n), and $A$ together with $\Sigma$ leads by law to $C$.

Suppose now that for a given counterfactual $A>C$ a set $\Gamma$ satisfies the conditions (1) $-(n)$ and together with $A$ leads by law to a $C^{\prime}$ incompatible with $C$. Then, using Lindenbaum's construction, one can extend $\Gamma$ to a $\Sigma$ that is maximal with respect to (1) $-(n)$ and which, given its compatibility with $A$, will not together with $A$ lead by law to $C$. Thus, (5.1) is at least as strong as an analogue of (1.4) using (1)-(n).

How does (5.1) fare with respect to our previous problems? It is easy to see that the second and third problems are still with us, although one should take into account that (5.1) may bar a counterfactual $A>C$ not only via a $C^{\prime}$ incompatible with $C$, but also by the existence of a maximal $\Sigma$ that together with $A$ does not lead to $C$, whether or not it leads to a $C^{\prime}$ incompatible with $C$. The first problem can be met if one assumes that the set of all legitimate information for a counterfactual $A>C$ is a subset of every maximal set satisfying (1)-(n). For, if $A>C$ is actually true - and so, by an earlier assumption, $A$ together with all legitimate information leads by law to $C$ - then (5.1) is satisfied. But I see no good reason to suppose that the preceding assumption will be true. Whether or not it is will depend on $(1)-(n)$, of course, and for certain relatively weak conditions it is easy to show that it is. The weaker one makes (1)- $(n)$, however, the more pressing become the other problems. These can be taken care of by an additional assumption; namely, that for any sentence $S$ that is illegitimate for $A>C$, there is a maximal set with respect to (1)-(n) which does not include $S$. Then one can define the set of relevant conditions outright as the intersection of all maximal sets with respect to (1)-(n). But, again, I see no reason to suppose that this further assumption will be true. 
Although I do not claim to have shown that Goodman's idea of a consistency clause is necessarily wrong, I am inclined to conclude that the only promising approach to the problem of relevant conditions is to attempt a direct characterization.

\section{Cotenability}

It is now due time to consider Goodman's notion of cotenability. His definition was:

(6.1) $S$ is cotenable with $A$ if $\neg(A>\neg S)$.

That cotenability can be used to characterize counterfactual truth is undeniable, because a counterfactual $A>C$ is true if and only if it is not the case that $\neg C$ is cotenable with $A$. So, the two notions are trivially equivalent.

This point seems to have escaped attention because Goodman and many commentators were concerned with cotenability as a means for discriminating the legitimate from the illegitimate information. Goodman's suggested amendment to clause [a] is to require that $S$ be cotenable, rather than merely compatible, with $A$. This does not work, however, as can be seen by considering once more the counterfactuals (1.2) and (1.3). Assuming that both of these are actually false, it is clear that the sentences Goodman uses to show that they satisfy clause [a] are not merely compatible but also cotenable with the antecedent. In fact, even if all other compatibility conditions were changed to cotenability conditions [a] would still be satisfied.

Of course, although it has been assumed in discussions of cotenability that Goodman's strengthening of clause [a] would be enough, one can still appeal to clause [b]. The first objection I raised in section 5 can now be met, because if $A>C$ is actually true, then $\neg C$ cannot be cotenable with $A$, and, hence, an $S^{\prime}$ satisfying $\left(4^{\prime}\right)$ would not be cotenable with $A$. The other objections still apply, however. But it is not clear whether Goodman meant to keep clause $[\mathrm{b}]$; since he considered the notion of cotenability unacceptable anyway, he did not pursue the matter any further.

Cotenability has also been characterized in a different way; namely, for true $S$ :

(6.2) $S$ is cotenable with $A$ if $A>S$.

David Lewis (p. 70) mentions this formulation and argues that on his theory - i.e., using his own analysis of ' $>$ ' - we have:

(6.3) $A>C$ if and only if there is a true $S$ cotenable with $A$ such that $A \& S$ leads by law to $C$.

To this it may be objected that if $A>S$ is also analyzed along Goodman's lines, so that $A>S$ satisfies the conditions for $A>C$ in (6.3), and (6.3) does not run into an infinite regress, then (6.2) is definitely unsatisfactory because $A>C$ will only 
satisfy the conditions of (6.3) if $C$ follows by law from $A$ alone. For, on the given assumptions, if $A>C$ satisfies (6.3) then there is a finite sequence of true sentences $S_{1}, \ldots, S_{n}$, such that $A$ leads by law to $S_{n}, A \& S_{k}$ leads by law to $S_{k-1}(1<k \leq n)$, and $A \& S_{1}$ leads by law to $C$. On the basis of this argument Bennett (p. 390) concludes that the proper analysis of cotenability is given by (6.1), not (6.2).

The assumption that $A>S$ is analyzed along Goodman's lines contains an important catch, however. Since $S$ is true, $A>S$ is the semifactual: even if $A$ had been true, $S$ would still be true. Moreover, Goodman's analysis of a semifactual $A>S$ is precisely that $\neg(A>\neg S)$, where $A>\neg S$ is, of course, a counterfactual. So, for true $S$, which is the relevant case, (6.1) and (6.2) actually coincide. In fact, Goodman himself points out that cotenability cannot be defined as in (6.2) if $A>S$ is analized in the manner appropriate to counterfactuals. Unfortunately, however, Goodman's analysis of semifactuals is incorrect. What is interesting is that although Goodman gets the right notion of cotenability as such, that is not what he wants. Let me explain.

Following my intuitions of cotenability, I would say that for any sentences $P$ and $Q$,

(6.4) $P$ and $Q$ are cotenable (jointly tenable) if neither $Q>\neg P$ nor $P>\neg Q$,

where ' $>$ ' is interpreted in the strong sense appropriate to counterfactuals. If one holds that ' $>$ ' satisfies contraposition, (6.4) boils down to (6.1). Otherwise, one can think of (6.1) as involving the asymmetrical relation $P$ is tenable relative to $Q$ characterized by the first conjunct of (6.4). At any rate, whether one talks of cotenability or relative tenability, it seems clear that Goodman's approach to this notion is intuitively correct independently of the truth-values of $P$ and $Q$. (6.2) on the other hand, although implying cotenability (with the proper analysis of ' $>$ ' as a counterfactual), is not a good analysis of cotenability as such. That is why the proviso that $S$ be true is crucial, for, if $S$ is false, the condition is much too strong. But even for a semifactual, (6.2) is not equivalent to cotenability. To see this we must realize where Goodman went wrong in his analysis of semifactuals.

The semifactual

even if $A$ had been the case, $S$ would still be the case,

is true if, and only if,

it is not the case that $S$ might not have been the case if $A$ had been the case.

In other words (Lewis, p. 21), a semifactual involves the denial of a "might" counterfactual and not, as Goodman supposes, of a "would" counterfactual. For example, if you and I went to a party, and I assert

Principia 15(3): 383-397 (2011). 
even if I hadn't gone, you would still have gone,

I mean to deny that you might not have gone if I hadn't gone.

So, the semifactual $A>S$ implies that $S$ is tenable relative to $A$, in Goodman's sense, but not vice-versa.

The objections to (6.2) and (6.3) now break down, but we are still left with the important problem that an appeal to "might" counterfactuals in our characterization of "would" counterfactuals begs the question in a very strong sense. For, $A>C$ if and only if $\neg(A \diamond>\neg C)$ gives us a perfectly good definition without the detour through relevant conditions.

There is, however, another characterization of the truth-conditions for semifactuals from which one can extract a more useful condition to be placed on the relevant conditions. Consider again the semifactual about the party. I suggest that we can distinguish two cases: either (i) the fact that you went to the party is independent of my going to the party - i.e., that I went was irrelevant to your going - or (ii) there are relevant considerations that establish that you would have gone even if I hadn't. The first case would apply if, for example, you didn't know that I was going and you went because you were invited by the hosts. The second case would apply if you went because I invited you to go with me but you had also been invited by another friend and, had I not gone, you would have gone with him. One has to be careful, of course, to fill in the details, so that, for example, the party's taking place was not affected by my (not) going, etc. I further suggest that what is important for the relevant conditions is that they be independent in the sense of case (i).

More specifically, my suggestion is that the desired version of (6.3) is:

(6.5) $A>C$ is true if there is a true $S$ independent of the truth of $\neg A$ (or of $A$ ) such that $A \& S$ leads by law to $C$.

Provided that ' $>$ ' is interpreted as a "would" conditional, (6.5) applies not only to actual counterfactuals but also to semifactuals and to "would" conditionals with true antecedent and true consequent. The full fledged semifactual used in (6.3) is not needed because, by an argument similar to Bennett's, if it were used it could be eliminated by further iterations of (6.5).

This notion of independence has appeared before in our considerations; for example, when I suggested at the end of section II that the reason Parry's $A \rightarrow C$ is illegitimate is that its truth depends on the truth of $\neg A$. Goodman's and Parry's suggestions discussed in sections 3 and 4 were attempts to capture something like the notion of independence. Similarly, Goodman's idea of cotenability seems to me another attempt in the same direction. I will now try to indicate briefly what I have in mind and how it differs from the other notions we considered so far. 


\section{Independence}

Independence, as I understand it, should not be confused with the related notion: $S$ is true whether or not $A$ is true. This can be understood as asserting that both $A>S$ and $\neg A>S$, or, equivalently, that neither $\neg A \diamond>\neg S$ nor $A \diamond>\neg S$. It is reasonable to say that if this is the case, then $S$ is true independently of whether or not $A$ is true. This is similar to another notion of cotenability discussed by Lewis and is obviously stronger than both Goodman's cotenability and of cotenability in the sense of (6.2). The notion of independence I have in mind is stronger still. The following example may illustrate the difference.

Suppose that Jones, wanting to kill himself, took a very strong poison at noon of a certain day. At $1 \mathrm{pm}$ nothing could have prevented his death shortly thereafter, but, at that time, Smith shot him and killed him. Now, it is clear that Jones would have died in the early afternoon whether or not Smith shot him at $1 \mathrm{pm}$. It is also clear that he died because Smith shot him. Alternatively, it is neither the case that Jones might not have died in the early afternoon if Smith hadn't shot him at $1 \mathrm{pm}$, nor that Jones might not have died in the early afternoon although Smith shot him at $1 \mathrm{pm}$. But, that Jones died in the early afternoon is not independent, in my sense, of Smith shooting him at $1 \mathrm{pm}$. If, on the other hand, Jones had already been dead at $1 \mathrm{pm}$, then Smith's shooting him would have made no difference, and Jones' death in the early afternoon would have been independent of Smith shooting him at $1 \mathrm{pm}$.

So, if $S$ and $A$ are true, the intuitive idea that $S$ is independent of $A$ can also be expressed by such locutions as: the truth of $A$ has no bearing on, or makes no difference for, or is irrelevant to, the truth of $S$. It is important to keep in mind, however, that these locutions refer to actual matters of fact, and connections between them, and not to possible or conceivable connections. If, in this example about Jones, he had survived a little longer, then his dying in the early afternoon might not have been independent of Smith's shooting him. But what we are assessing are the actual circumstances of the case.

Although there are many cases in which it is very difficult to say whether or not a truth is independent of another, I believe that our intuitions concerning this notion are reasonably clear. At least as clear, at any rate, as the notion of consequence by law. In fact, the two notions stand at opposite poles of the same spectrum. If $S$ follows by law from A alone, non-trivially, then we have the tightest of connections between them. If, on the other hand, $\mathrm{S}$ is independent of $\mathrm{A}$, then we have the loosest connection - i.e., no connection at all. Conditionals of various sorts are in between.

It is interesting to note that if we try to characterize independence in terms of counterfactuals or other conditionals, we run into similar sorts of problems as in our attempts to characterize counterfactuals in terms of consequence by law. ${ }^{1}$ 


\section{References}

Bennett, J. 1974. Counterfactuals and Possible Worlds. Canadian Journal of Philosophy 4: 381-402.

Chateaubriand, O. 2001. Logical Forms. Part I: Truth and Description. Coleção CLE, Unicamp.

- 2004. Counterfactuals: Reply to Claudio Pizzi. Manuscrito 27: 65-77.

2005. Logical Forms. Part II: Logic, Language, and Knowledge. Coleção CLE, Unicamp.

Goodman, N. 1947. The Problem of Counterfactual Conditionals. The Journal of Philosophy

44: 113-28. Reprinted in Fact, Fiction, and Forecast, Harvard University Press, 1955. Second edition, Bobbs-Merrill Co., 1965. References to the second edition.

- 1957. Parry on Counterfactuals. The Journal of Philosophy 54: 442-45.

Lewis, D. 1973. Counterfactuals. Harvard University Press.

Parry, W. T. 1957. Reexamination of the Problem of Counterfactual Conditionals. The Journal of Philosophy 54: 85-94.

Pizzi, C. 2004. Chateaubriand on the Ambiguity of Counterfactual Suppositions. Manuscrito 27: 55-64.

Oswaldo Chateaubriand

Pontifícia Universidade Católica do Rio de Janeiro

Centro de Teologia e Ciências Humanas

Departamento de Filosofia

Rua Marquês de São Vicente 225

Gávea 22453-900 - Rio de Janeiro, RJ

Brasil

ochateaubriand@gmail.com

Resumo. O artigo de Goodman "The Problem of Counterfactual Conditionals" teve um papel central no debate relativo a análise adequada dos condicionais contrafactuais. A seguir examinarei o artigo de Goodman em detalhe e discutirei algumas objeções e sugestões de Parry em seu artigo "A Reexamination of the Problem of Counterfactual Conditionals". Restringirei minha discussão ao "problema das condições relevantes", assim denominado por Goodman, que é o tema principal das críticas de Parry e que considero ser o problema principal para a abordagem de Goodman.

Palavras-chave: N. Goodman; W. T. Parry; condicionais contrafactuais; condições relevantes.

\section{Notes}

${ }^{1}$ A brief discussion of the issues taken up in this paper appears on p. 393-394 of my book Logical Forms. Part II. In my talk at the $7^{\text {th }}$ Principia Symposium I also discussed the question of the proper analysis of logical form for counterfactuals. I could not include this discussion here, but it was briefly mentioned on pp. 73-74 of Logical Forms. Part I, and it was the subject of an exchange with Claudio Pizzi in 2004.

Principia 15(3): 383-397 (2011). 